Hautarzt 2022 $\cdot 73: 156-159$

https://doi.org/10.1007/s00105-021-04931-0

Angenommen: 7. Dezember 2021

Online publiziert: 14. Dezember 2021

(c) The Author(s), under exclusive licence to Springer Medizin Verlag GmbH, ein Teil von Springer Nature 2021

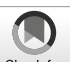

\section{Erythroderme Psoriasis nach COVID-19-Erkrankung}

\author{
Jeta Demiri - Miriam Abdo · Athanasios Tsianakas ${ }^{1}$ \\ 'Fachklinik Bad Bentheim, Bad Bentheim, Deutschland
}

\title{
Zusammenfassung
}

Wir präsentieren einen klinischen Fall eines Patienten mit akut exazerbierter erythrodermer Plaquepsoriasis nach einer symptomatischen Infektion mit SARSCoV-2. Es sind bereits unterschiedliche Faktoren bekannt, die zu einer Exazerbation der Psoriasis führen können wie Medikamente oder Infektionen mit z. B. Streptokokken. Ein Zusammenhang zwischen Psoriasis und einer Infektion mit dem neuartigen Coronavirus SARS-CoV-2 wurde v. a. in Fallberichten beschrieben, in denen eine medikamentöse Behandlung mit z. B. Hydroxychloroquin erfolgt war, ein bekannter Trigger der Psoriasis. In der Folge gab es auch einzelne Beschreibungen einer Exazerbation nach COVID-19 ohne medikamentösen Trigger, teils auch als pustulöse Psoriasisform. Unser Artikel zeigt erstmals einen Fall einer Psoriasiserythrodermie getriggert durch eine COVID-19Erkrankung ohne offensichtlich zusätzlich medikamentösen Auslöser.

\section{Schlüsselwörter}

Psoriasis vulgaris · SARS-CoV-2 $\cdot$ Exazerbation $\cdot$ Trigger $\cdot$ IL-17

\section{Anmerkung}

Aus Gründen der besseren Lesbarkeit wird auf die gleichzeitige Verwendung der Sprachformen männlich, weiblich und divers $(\mathrm{m} / \mathrm{w} / \mathrm{d})$ verzichtet. Sämtliche Personenbezeichnungen gelten gleichermaßen für alle Geschlechter.

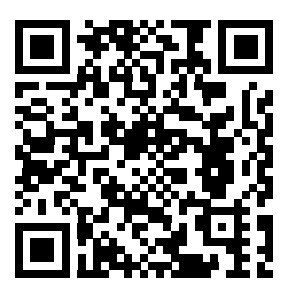

QR-Code scannen \& Beitrag online lesen
Psoriasis ist eine immunvermittelte chronisch entzündliche Hauterkrankung, die jede Hautoberfläche betreffen kann und sich meist als erythematöse, schuppende Plaques darstellt [1]. Die Prävalenz der Psoriasis liegt weltweit bei ca. 2\% [1]. Es gibt mehrere bekannte Faktoren, die eine Psoriasisexazerbation triggern können. Neben Nikotin, Alkohol, einem erhöhten Body Mass Index (BMI), Stress, Traumata und Infektionen gehören bestimmte Medikamente, wie z. B. $\beta$-Blocker, Lithium, Antimalariamedikamente (Hydroxychloroquin), Interferone, Imiquimod, ACE-Hemmer, Terbinafin, Tetracyclin, NSARs und Fibrate, dazu [2-4].

Der Mechanismus der Beeinflussung durch Medikamente ist komplex. Es ist jedoch bekannt, dass einige Arzneimittel die Hyperproliferation von Keratinozyten und die IL-23/IL-17-Achse beeinflussen können [4]. $\beta$-Blocker führen zu einer Abnahme des intraepidermalen zyklischen Adenosinmonophosphats (cAMP), ein intrazellulärer Botenstoff, der für die Stimulation von Proteinen zur zellulären Differenzierung und Hemmung der Proliferation ver- antwortlich ist, und verursachen dadurch eine Hyperproliferation der Keratinozyten $[2,3]$. Ein Zusammenhang zwischen einer Exazerbation der Psoriasis und einer Infektion mit beispielsweise Streptokokken, Staphylococcus aureus oder einer HIV-Infektion wurde bereits belegt $[4,5]$.

Ein Zusammenhang zwischen Psoriasis vulgaris und einer Infektion mit dem neuartigen Coronavirus SARS-CoV-2 (COVID-19) wurde bereits kurz nach Ausbruch der Pandemie beschrieben. Hierbei fiel auf, dass in allen anfänglichen Fallberichten die Patienten das Antimalariamedikament Hydroxychloroquin erhalten hatten, was ein bekannter medikamentöser Trigger der Psoriasis ist $[6-8,24]$. Mechanistisch liegt der Exazerbation das inhibitorische Potenzial von Hydroxychloroquin auf die epidermale Transglutaminase zugrunde [27, 28]. Dies bedingt eine unkontrollierte Keratinozytenhyperproliferation, was sogar in seltenen Fällen eine De-novo-Psoriasis pustulosa auslösen kann [3].

Später wurden in einzelnen Fällen nach COVID-19-Erkrankung auch ohne medikamentösen Trigger das Auftreten einer Pso- 


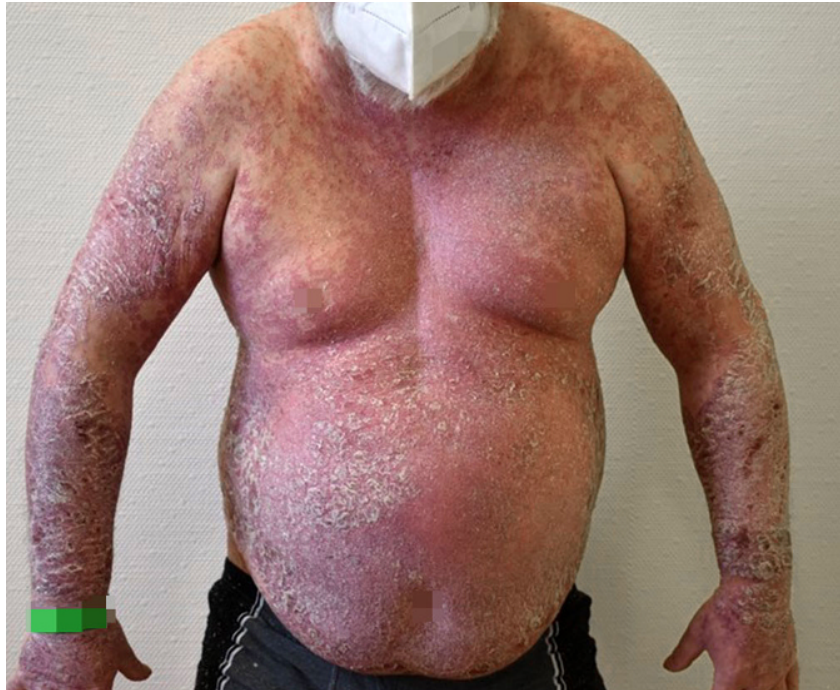

Abb. 1 ム Exazerbation der Psoriasis vulgaris nach Infektion mit SARS-CoV-2, Erythrodermie mit ausgeprägten großfächig konfluierenden erythematosquamösen Plaques

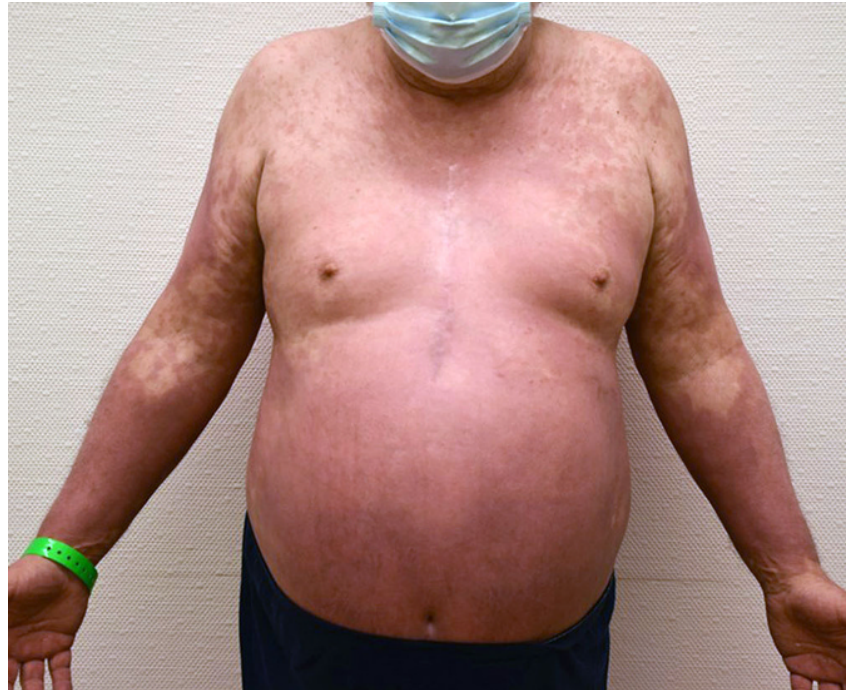

Abb. $2 \Delta$ Verbesserung der Psoriasisläsionen nach 14 Tagen Therapie riasis guttata sowiez. B. der Übergang einer Psoriasis pustulosa vom Typ Hallopeau in eine generalisierte pustulöse Psoriasis beschrieben $[9,26,30]$. Dass COVID-19, wie im klinischen Alltag oft beobachtet, eine bekannte Psoriasis verschlechtern kann, wurde kürzlich mittels einer multizentrischen Beobachtungsstudie publiziert, in der es bei 23 der 358 beobachteten Psoriasispatienten $(6,4 \%)$ zu COVID-19 gekommen war [25]. In 15 dieser 23 Fälle $(65,2 \%)$ wurde eine Exazerbation der Psoriasis beschrieben, jedoch in keinem Fall eine Erythrodermie. Wie hoch der Anteil der Patienten mit Hydroxychloroquin-Einnahme lag, wurde nicht beschrieben. Somit stellt unser unten beschriebener Fall die Erstbeschreibung einer Erythrodermie nach COVID-19 bei vorbekannter Psoriasis dar.

Der Mechanismus der Immunantwort auf COVID-19 ist sehr komplex und hängt hauptsächlich von B- und T-Zellen, NKZellen wie auch mehreren Mediatoren wie IL-12, IL-15, Interferon- $\alpha / \beta$ und $-\gamma$ ab [10]. Bei schweren Verläufen wurde bei einer Gruppe von COVID-19-Erkrankten auch eine Erhöhung proinflammatorischer Zytokine wie IL-6, TNF- $a$, IL-17A beobachtet, die bekannte Ziele von Psoriasissystemtherapeutika sind $[10,11]$. Italienische Studien konnten aber keine eindeutigen Signale für schwerere Verläufe der COVID19-Erkrankung bei Psoriasis zeigen [10]. Der Nachweis von SARS-CoV-2 erfolgt re- gelhaft mittels PCR-Untersuchung eines Nasopharyngealabstrichs. Beim Auftreten von Hautmanifestationen im Rahmen der Erkrankung gelang der PCR-Virusnachweis vor Kurzem sogar in einer Hautprobe [12].

Im Rahmen des Fortschreitens der Impfkampagne gegen COVID-19 sehen wir im klinischen Alltag zahlreiche exazerbierte Psoriasispatienten auch nach Vakzinierung unabhängig von der Art des Vakzins (mRNA- oder Vektor-basiert). Dies wurde erst kürzlich in einer Fallserie bestätigt [29].

\section{Fallbeschreibung}

Anamnese. Wir berichten hier über einen 60 Jahre alten Patienten mit einer seit 1998 bekannten Psoriasis vulgaris, der sich nach einer durchgemachten symptomatischen Infektion mit SARS-CoV-2 mit positivem und nach häuslicher Quarantäne negativem Nachweis durch PCR-Abstrich in unserer Klinik vorstellte. Bereits 2018 sei es nach einer bakteriell verursachten Pneumonie zu einer Exazerbation der Psoriasis gekommen. Neben einer mittelschweren Psoriasis vulgaris waren bei dem Patienten ein Zustand nach Myokardinfarkt 2018, eine mechanische Aortenklappe, ein metabolisches Syndrom mit arterieller Hypertonie, Diabetes mellitus Typ II, Hypercholesterinämie und Adipositas (BMI 35,2 bei $173 \mathrm{~cm}$ Körpergröße und $104 \mathrm{~kg}$ Körper- gewicht) sowie eine COPD (Z.n. ca. 100 Packyears) bekannt. Der Patient erkrankte im Januar 2021 an COVID-19. Neben starker Abgeschlagenheit entwickelte er im Verlauf der Erkrankung Geschmacks- und Geruchsverlust, Appetitlosigkeit sowie Kopfschmerzen. Laut Patient seien kein Fieber, Husten oder stärkere Luftnot aufgetreten, sodass lediglich eine häusliche Quarantäne über 14 Tage ohne neue Medikation oder Änderung der Dauermedikation mit anschließend negativem Abstrich erfolgte. Fünf Tage nach Nachweis der Infektion kam es zu einer massiven Exazerbation mit Erythrodermie der zuletzt unter Lokaltherapie über etwa 6 Monate gut kontrollierten Psoriasis (vormals nur leichtgradige Herde an Ellenbogen und Knie, PASI <3). In der Vergangenheit habe es bei stärkerer Ausprägung Therapieversuche mit Fumarsäureestern und Acitretin über jeweils 4 Wochen gegeben, die bei Unverträglichkeit abgesetzt werden mussten. Zuletzt sei eine systemische Therapie mit MTX $20 \mathrm{mg}$ s.c. von $02 / 19$ bis Sommer 2020 erfolgt, die bei gutem Hautbefund vom Patienten eigenständig abgesetzt worden war (danach keine Verschlechterung).

Befund. Bei der Vorstellung zeigte der Patient eine Erythrodermie mit ausgeprägten konfluierenden erythematosquamösen Plaques am ganzen Integument mit besonderer Betonung des Stammes 
und der Extremitätenstreckseiten (PASI 57,6) (• Abb. 1). Durch die vorhandenen Hautschmerzen war der Patient in seiner Bewegung stark eingeschränkt.

Therapie und Verlauf. Es erfolgten die stationäre Aufnahme des Patienten und Behandlung mit Balneophototherapie (initial Schwefelmineralwasser, im Verlauf Solebäder + UVB $311 \mathrm{~nm}$ ) und Lokalsteroiden. Im Verlauf wurde nach durchgemachter Infektion mit SARS-CoV-2 die systemische Therapie mit MTX $15 \mathrm{mg}$ 1-mal/ Woche s.c. wieder eingeleitet. Dies erfolgte aufgrund des in der Vergangenheit guten Therapieansprechens unseres Patienten auf MTX und der bis dato guten Sicherheitslage von MTX im Fall des Auftretens von COVID-19 (keine schlechteren COVID19-Verläufe bei MTX-Patienten beschrieben [31, 32]). Die Laboruntersuchungen bei Aufnahme zeigten: CRP 2,75 mg/dl (normal: $<0,5 \mathrm{mg} / \mathrm{dl}$ ), BSG $30 \mathrm{~mm} / \mathrm{h}$ (normal: $<20 \mathrm{~mm} / \mathrm{h}$ ), Leukozyten 11,59 Tsd/ $\mu \mathrm{l}$ (normal:3,91-10,90 Tsd/ $\mu \mathrm{l}$ ), NeutrophilenLymphozyten-Ratio (NLR) 5,01. Unter der Therapie konnte innerhalb von 14 Tagen eine deutliche Besserung des Hautbefundes erzielt werden (PASI 25,6) (• Abb. 2). Die Laborkontrolle nach 3 Tagen zeigten bereits einen deutlichen Rückgang der Entzündungsparameter: CRP $0,97 \mathrm{mg} / \mathrm{dl}$ (normal: $<0,5 \mathrm{mg} / \mathrm{dl}$ ), BSG $11 \mathrm{~mm} / \mathrm{h}$ (normal: $<20 \mathrm{~mm} / \mathrm{h}$ ), Leukozyten 7,48Tsd/ $\mu \mathrm{l}$ (normal: 3,91-10,90 Tsd/ $\mu \mathrm{l}$ ), NeutrophilenLymphozyten-Ratio (NLR) 3,48.

\section{Diskussion}

Unser Fall stellt die erste Publikation einer Psoriasiserythrodermie nach COVID-19-Erkrankung ohne medikamentösen Trigger dar. Die beschriebene Exazerbation der Psoriasis bei unserem vorgestellten Patienten unterstützt die aus Fallberichten und einer multizentrischen Beobachtungsstudie bekannte Hypothese, dass eine COVID19-Infektion einen starken Trigger für eine Psoriasis darstellt $[9,25]$. Da während einer COVID-19-Erkrankung bekanntermaßen stark erhöhte Spiegel u.a. der Psoriasisschlüsselzytokine IL-17 oder auch TNF-a gemessen werden können, mag dies eine Erklärung für eine derartige Exazerbati- on sein [13]. Eine spezifische antipsoriatische Therapie wie die Balneophototherapie kombiniert mit einer Systemtherapie wie Methotrexat vermag, wie berichtet, diese Exazerbation jedoch wieder erfolgreich zu therapieren.

Ob eine Systemtherapie den Verlauf einer SARS-CoV-2-Erkrankung negativ beeinflusst, bleibt bis heute umstritten. Die Psoriasis stellt selbst ggf. einen Risikofaktor für symptomatische COVID-19-Verläufe dar [14]. In der Literatur finden sich zahlreiche Übersichtsartikel, Fallserien und retrospektive Untersuchungen, die bislang aber nicht eindeutig zeigen konnten, dass eine Systemtherapie einen Risikofaktor für schlechtere klinische Verläufe von COVID19 darstellt [15-18].

Es wird lediglich auf Grundlage allgemeiner Sicherheitsprofile aus Registern aus der Vor-Corona-Zeit geschlussfolgert, dass Präparate wie Ciclosporin, Methotrexat oder TNF-a-Inhibitoren einen SARSCoV-2-infektionsfördernden Effekt haben könnten. Für MTX hingegen zeigen aktuelle Registerdaten, dass es in der Realität hier zu keinen schlechteren Verläufen gekommen ist [31, 32]. Ferner könnten moderne Biologika wie IL-17- oder IL23-Antikörper protektive Effekte besitzen, da sie den bei COVID-19 kritischen Zytokinsturm abmildern könnten [10, 11]. Dies beruht jedoch nur auf theoretischen Modellen. Die bis dato größte Analyse von Verläufen von COVID-19 bei Psoriasispatienten wurde kürzlich auf dem Jahreskongress der Amerikanischen Dermatologischen Gesellschaft AAD (Annual Congress of the American Academy of Dermatology) vorgestellt [19]. Hier wurden Daten einer der größten Gesundheitsdatensätze der USA (Symphony Dataset) präsentiert und bei mehr als 167.000 Psoriasispatienten das Auftreten von COVID19-Erkrankungen sowie deren Korrelation mit topischen Systemtherapeutika gezeigt (>36.000 Datensätze von mit Biologika behandelten Psoriasispatienten). Es zeigte sich, dass Psoriasispatienten im Vergleich zu Kontrollpersonen eine um 33\% erhöhte Inzidenz an COVID-19 aufwiesen. Eine Behandlung mit TNF-a-Inhibitoren führte jedoch zu einer um $18 \%$ reduzierten Inzidenz gegenüber rein topisch behandelten Psoriasispatienten bzw. Reduktion um $17 \%$ gegenüber Kontrollen.
Unter Methotrexat kam es sogar zu einer Inzidenzreduktion gegenüber topisch behandelten Psoriasispatienten um 25\%. Unter Behandlung mit IL-17-Inhibitoren stieg die Inzidenz gegenüber Kontrollen um 51 und bei Therapie mit dem IL12/23-Antikörper Ustekinumab um 37\% an. Dies mag dadurch zu erklären sein, dass IL-17 und IL-23 bei der initialen Abwehr einer Infektion mit SARS-CoV-2 eine wichtige Rolle spielen. Zum Thema Hospitalisierung und Mortalität wurde kürzlich ein Review-Artikel publiziert, der sämtliche Publikationen bis zum 09.06.2020 in MEDLINE (PubMed) zu den Schlagwörtern "Psoriasis, Biologics und COVID-19" auswertete. Hierbei zeigte sich, dass etwa 0,3\% der Psoriasispatienten unter einer COVID-19-Erkrankung litten, wobei die Hospitalisierungsrate bei $0,1 \%$ lag. Unter den insgesamt 10.509 Patienten wurde kein Tod durch COVID-19 gemeldet [20].

Zu einer Exazerbation einer bestehenden Psoriasis kann es aber auch nach (COVID-19-)Impfungen kommen [30]. Dass Impfungen zur Exazerbation einer Psoriasis führen können, ist z. B. für die Influenzaimpfung bekannt [21]. Exazerbationen nach COVID-19-Vakzinierung konnten wir bei zahlreichen unserer Patienten mit Psoriasis beobachten. Trotz allem gilt für unsere Patienten die dringende Empfehlung für eine Durchführung der Impfung, um schwere klinische Verläufe von COVID-19 zu verhindern. Sowohl von der Deutschen Dermatologischen Gesellschaft als auch der Deutschen Gesellschaft für Rheumatologie gibt es keine klare Empfehlung, eine immunmodulierende Therapie zu verändern bzw. mehrwöchige Therapiepausen zu implementieren (eine Ausnahme stellt hier lediglich der B-Zelldepletierende Antikörper Rituximab dar) $[22,23]$. Ob Präparate, wie z.B. Methotrexat, um den Zeitpunkt der Impfung herum pausiert werden sollen, ist nach Studienlage nicht klar zu sagen, sodass der betreuende Arzt hier die Entscheidung zu treffen hat [23].

Fazit für die Praxis

- Eine COVID-19-Erkrankung, aber auch eine Vakzinierung gegen COVID-19 können zu den neuen Triggerfaktoren der Psoriasis gezählt werden. Im Umkehrschluss bedeutet dies, dass bei der Betreuung von 
Psoriasispatienten bei aktiver COVID-19Erkrankung bzw. nach Impfung eine erhöhte Aufmerksamkeit herrschen sollte, um etwaige Exazerbationen umgehend zu therapieren und somit stärkste Exazerbationen wie eine Erythrodermie zu verhindern.

- Durch eine adäquate antipsoriatische Therapie kann eine mögliche Exazerbation schnell und erfolgreich therapiert werden, sodass das Exazerbationsrisiko kein Argument gegen eine Vakzinierung darstellt.

Korrespondenzadresse

PD Dr. med. Athanasios Tsianakas

Fachklinik Bad Bentheim

Am Bade 1, 48455 Bad Bentheim, Deutschland a.tsianakas@fk-bentheim.de

\section{Einhaltung ethischer Richtlinien}

Interessenkonflikt. J. Demiri, M. Abdo und A. Tsianakas geben an, dass kein Interessenkonflikt besteht.

Für diesen Beitrag wurden von den Autoren keine Studien an Menschen oder Tieren durchgeführt. Für die aufgeführten Studien gelten die jeweils dort angegebenen ethischen Richtlinien. Für Bildmaterial oder anderweitige Angaben innerhalb des Manuskripts, über die Patienten zu identifizieren sind, liegt von innen und/oder ihren gesetzlichen Vertretern eine schriftliche Einwilligung vor.

\section{Literatur}

1. Rendon A, Schäkel K (2019) Psoriasis pathogenesis and treatment. Int J Mol Sci 20(6):1475

2. Balak DM, Hajdarbegovic E (2017) Drug-induced psoriasis: clinical perspectives. Psoriasis 7:87-94

3. Kim GK, Del Rosso JQ (2010) Drug-provoked psoriasis: Is it drug induced or drug aggravated?: Understanding pathophysiology and clinical relevance. J Clin Aesthet Dermatol 3:32-38

4. Visser MJE, Kell DB, Pretorius E (2019) Bacterial dysbiosis and translocation in psoriasis vulgaris. Front Cell Infect Microbiol 9:7

5. Mallon E, Bunker CB (2000) HIV-associated psoriasis. AIDS Patient Care STDS 14:239-246

6. Kutlu Ö, Metin A (2020) A case of exacerbation of psoriasis after oseltamivir and hydroxychloroquine in a patient with COVID-19: Will cases of psoriasis increase after COVID-19 pandemic? Dermatol Ther 33(4):e13383

7. Ozaras R, Berk A, Ucar DH et al (2020) Covid19 and exacerbation of psoriasis. Dermatol Ther 33(4):e13632

8. Nasiri S, Araghi F, Tabary M et al (2020) A challenging case of psoriasis flare-up after COVID19 infection. J Dermatolog Treat 31(5):448-449

9. Elrazi A, Abdelaziz M, Joud A et al (2021) SARSCoV-2 and guttate psoriasis: a case report and review of literature. Clin Case Rep 9(7):e4568

10. Buhl T, Beissert S, Gaffal E et al (2020) COVID19 und Auswirkungen auf dermatologische und

\section{Erythrodermic psoriasis after COVID-19}

We present a clinical case of a patient with acutely exacerbated erythrodermic psoriasis vulgaris after symptomatic infection with SARS-CoV-2 (severe acute respiratory syndrome coronavirus 2). Various factors are already known that can lead to an exacerbation of psoriasis, such as drugs or infections with, for example, streptococcus. An association between psoriasis and an infection with SARS-CoV-2 has been described so far in individual case reports, in which, however, drug treatment with for example hydroxychloroquine, a known trigger of psoriasis, often took place. Later cases of exacerbation of psoriasis, partly as pustular psoriasis have been published also without drug induction. However we present for the first time a case of erythrodermic psoriasis triggered by COVID-19 (coronavirus disease 2019) without an obvious drug trigger.

\section{Keywords}

Psoriasis vulgaris $\cdot$ SARS-CoV-2 $\cdot$ Exacerbation $\cdot$ Trigger $\cdot \mathrm{IL}-17$

allergologische Erkrankungen. J Dtsch Dermatol Ges 18(8):815-825

11. Buszko M, Nita-Lazar A, Park J-H et al (2021) (2021) Lessons learned: new insights on the role of cytokines in COVID-19. Nat Immunol 22(4):404-411

12. Jamiolkowski $D$, Mühleisen $B$, Müller $S$ et al (2020) SARS-CoV-2 PCR testing of skin for COVID-19 diagnostics: a case report. Lancet 396(10251):598-599

13. Ragab D, Salah Eldin H, Taeimah M et al (2020) The COVID-19 Cytokine storm; what we know so far. Front Immunol 11:1446

14. Kutlu Ö, Metin A (2020) Dermatological diseases presented before COVID-19: Are patients with psoriasis and superficial fungal infections more vulnerable to the COVID-19? Dermatol Ther 33:e13509

15. Amerio P, Prignano F, Giuliani F et al (2020) COVID 19 and psoriasis: Should we fear for patients treated with biologics? Dermatol Ther 33:e13434

16. Elmas ÖF, Demirbaş A, Kutlu Ö et al (2020) Psoriasis and COVID-19: A narrative review with treatment considerations. Dermatol Ther 33(6):e13858

17. Mahil SK, Dand N, Mason KJ et al (2021) Factors associated with adverse COVID-19 outcomes in patients with psoriasis-insights from a global registry-based study. J Allergy Clin Immunol 147(1):60-71

18. de Wijs LEM, Joustra MM, Olydam Jl et al (2021) COVID-19 in patients with cutaneous immunemediated diseases in The Netherlands: real-world observational data. J Eur Acad Dermatol Venereol 35(3):e173-e176

19. Wu JJ, Liu J, Thatiparthi A et al (2021) Association between systemic treatments and COVID-19 infection risk in patients with psoriasis. https://www. aad.org/member/meetings-education/aadvmx (Virtueller Kongress der American Academy of Dermatology, Late Breaker Session 24.04.2021). Zugegriffen:22. Juni 2021

20. Ebrahimi A, Sayad B, Rahimi Z (2020) COVID-19 and psoriasis: biologic treatment and challenges. JDermatolog Treat 6:1-5.https://doi.org/10.1080/ 09546634.2020.1789051 (PMID: 32598204)

21. Munguía-CalzadaP,Drake-MonfortM,ArmestoSet al (2020) Psoriasis flare after influenza vaccination in Covid-19 era: a report of four cases from a single center. Dermatol Ther 34(1):e14684

22. Stellungnahme der Deutschen Dermatologischen Gesellschaft zum Thema COVID-19 Vakzinierung unter Systemtherapie. https://derma.de/corona/
impfung-gegen-sars-cov-2/.Zugegriffen: 13. Dez. 2021

23. Stellungnahme der Deutschen Dermatologischen Gesellschaft zum Thema COVID-19 Vakzinierung unter Systemtherapie. https://dgrh.de/ Start/Wissenschaft/Forschung/COVID-19.html. Zugegriffen: 13.Dez. 2021

24. Sachdeva M, Mufti A, Maliyar K et al (2020) Hydroxychloroquine effects on psoriasis: $A$ systematic review and a cautionary note for COVID19 treatment. J Am Acad Dermatol 83(2):579-586

25. Kara Polat A, Oguz Topal I, Karadag AS et al (2021) The impact of COVID-19 in patients with psoriasis: a multicenter study in Istanbul. Dermatol Ther 34(1):e14691

26. Gananandan K, Sacks B, Ewing I (2020) Guttate psoriasis secondary to COVID-19. BMJ Case Rep 13(8):e237367

27. Gravani A, Gaitanis G, Zioga A et al (2014) Synthetic antimalarial drugs and the triggering of psoriasis do we need disease-specific guidelines for the management of patients with psoriasis at risk of malaria? Int J Dermatol 53(3):327-330

28. Said A, Bock S, Lajqi T et al (2014) Chloroquine promotes IL-17 production by CD41 T cells via $\mathrm{p} 38$ - dependent IL-23 release by monocyte-derived Langerhanslike cells. J Immunol 193(12):6135-6143

29. Sotiriou E, Tsentemeidou A, Bakirtzi K et al (2021) Psoriasis exacerbation after COVID-19 vaccination: a report of 14 cases from a single centre. J Eur Acad Dermatol Venereol. https://doi.org/10.1111/jdv. 17582

30. Samotij D, Gawron E, Szczech J et al (2021) Acrodermatitis continua of Hallopeau evolving into generalized pustular psoriasis following COVID-19: a case report of a successful treatment with Infliximab in combination with Acitretin. Biologics 27(15):107-113

31. Gianfrancesco M, Hyrich KL, Al-Adely S et al (2020) Characteristics associated with hospitalisation for COVID-19 in people with rheumatic disease: data from the COVID-19 Global Rheumatology Alliance physician-reported registry. Ann Rheum Dis 79(7):859-866

32. FAI2R/SFR/SNFMI/SOFREMIP/CRI/IMIDIATE consortium and contributors (2020) Severity of COVID19 and survival in patients with rheumatic and inflammatory diseases: data from the French RMD COVID-19 cohort of 694 patients. Ann Rheum Dis 80(4):527-538 